\title{
EVIDENCE OF A LOWER MANTLE SHEAR VELOCITY DISCONTINUITY IN S AND SS PHASES
}

\author{
Thorne Lay
}

\author{
Department of Geological Sciences, University of Michigan
}

\begin{abstract}
Teleseismic recordings of direct $\mathrm{S}$ and $\mathrm{sS}$ body wave phases, and their core-reflected counterparts ScS and sScS, from intermediate and deep focus earthquakes are used to analyze the lowermost mantle shear velocity structure beneath Alaska. A model with a $2.75 \%$ shear velocity increase $280 \mathrm{~km}$ above the core-mantle boundary accurately matches waveform complexities in both the $\mathrm{S}$ and sS wavetrains. Variations in source depth produce systematic shifts in the timing of the triplication arrivals between the $S$ and sS travel time branches that are readily observed in long-period WWSSN tangential component recordings. The systematic range- and depth-dependence of the observed shifts are well-predicted by the discontinuity model, and preclude explanations of the waveform complexity as resulting from multiple ruptures at the source, receiver reverberations, or near-source scattering from slab structure.
\end{abstract}

\section{Introduction}

Accurate models of the seismic velocity structure in the lowermost $200 \mathrm{~km}$ of the mantle (D" region) are essential for calculations of the thermal history and dynamics of the core and mantle. Several recent investigations of seismic body waves have indicated the presence of velocity stratification above the core-mantle boundary [Lay and Helmberger, 1983; Wright et al., 1985], while models of long-wavelength lateral velocity variations in the lower mantle indicate strong heterogeneities in the D" region [Dziewonski, 1984; Clayton and Comer, 1983]. Current thermal models indicate a large temperature contrast of about $1000^{\circ}$ between the core and mantle, requiring a thermal boundary layer at the base of the mantle [Jeanloz and Richter, 1979; Loper, 1984]. This is consistent with the general decrease in velocity gradients observed in the lowermost mantle [Doornbos, 1983; Doornbos et al., 1986]. The combined effects of stratification, lateral heterogeneity, and decreased velocity gradients complicate interpretation of seismic data sampling the D" region. It is particularly important to resolve whether velocity discontinuities are actually present within D", since compositional stratification would strongly affect the thermal boundary layer dynamics, as well as the interpretation of the lateral velocity variations. This paper presents an analysis of a combination of seismic body waves that is particularly sensitive to the presence of a velocity discontinuity in D".

Lay and Helmberger [1983] found evidence for a $2.75 \%$ shear velocity discontinuity about $280 \mathrm{~km}$ above the core in $S$ waves from intermediate and deep focus earthquakes. The long-period tangential component seismograms they analyzed show waveform complexities in the distance range $75^{\circ}$ to $95^{\circ}$ that were attributed to a triplication produced by an abrupt velocity increase. This interpretation was criticized by Schlittenhardt et al. [1985], who argue that such a discontinuity is incompatible with

Copyright 1986 by the American Geophysical Union.

Paper number 6L6991.

0094-8276/86/006L-6991\$03.00 observed signals that diffract around the core. Lay [1985] showed that this objection could be eliminated by slight modifications of the proposed velocity models, while retaining the velocity discontinuity needed to match the undiffracted observations. One of the strongest tests of the discontinuity model is to establish whether it is consistent with other seismic phases that sample the D" region. The surface-reflected sS phases from intermediate and deep focus events, which, like direct $S$ phases, sample the deep mantle at large epicentral distances (Figure 1), are radiated upward from the sources and thus have very different near-source paths than the downgoing direct $S$ phases. If lower mantle velocity stratification is responsible for the waveform complexities that Lay and Helmberger [1983] have observed in profiles of direct $S$ waves, corresponding complexities should be observed in the sS phases from the same events. This paper will establish that the sS phases support the discontinuity model.

\section{Data Analysis}

A comparison of direct $S$ and the surface reflection $\mathrm{s} S$ phases has three important attributes for interrogating deep mantle structure. The sS phases should encounter the same deep mantle structure as direct $S$, providing a test of the deep discontinuity model. Secondly, the depth of the source influences the relative timing of the $\mathrm{S}$ and sS travel time branches, as shown in Figure 2. For a surface focus event the branches will exactly overlie. With increasing focal depth, the branches separate systematically, with the $\mathrm{sS}$ branch resembling that of direct $\mathrm{S}$ for a surface focus event at closer distance (see Figure 1). For the lower mantle discontinuity model, SLHO, of Lay and Helmberger [1983], the entire triplication curve for sS should shift toward greater distances relative to that of direct $S$ as the source depth increases. Figure 2 indicates that the shift of the crossover distance is minor for a $105 \mathrm{~km}$ deep event, but is an easily observable $6^{\circ}$ for a $600 \mathrm{~km}$ deep event. For a given seismic recording from a $100 \mathrm{~km}$ deep

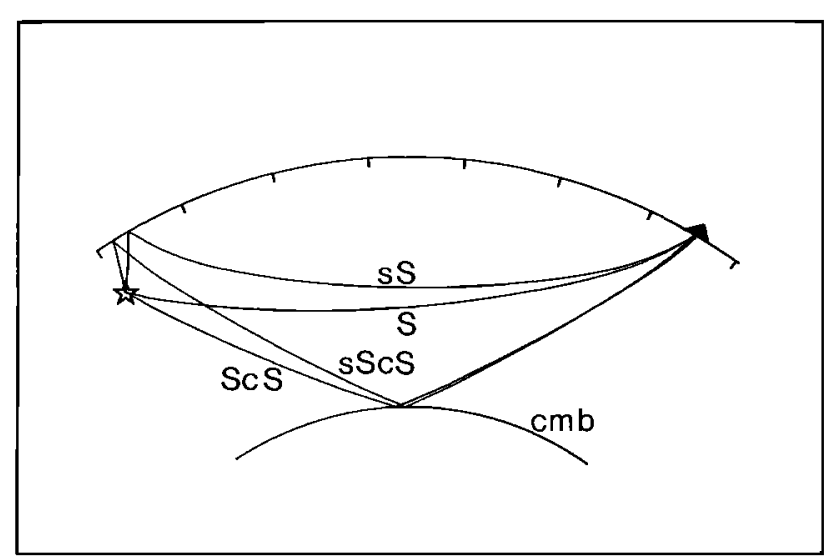

Fig. 1. Raypaths for $S$ and sS phases from a $600 \mathrm{~km}$ deep event to a station $65^{\circ}$ away. The core-reflections $\mathrm{ScS}$ and $\mathrm{sScS}$ are also shown. 


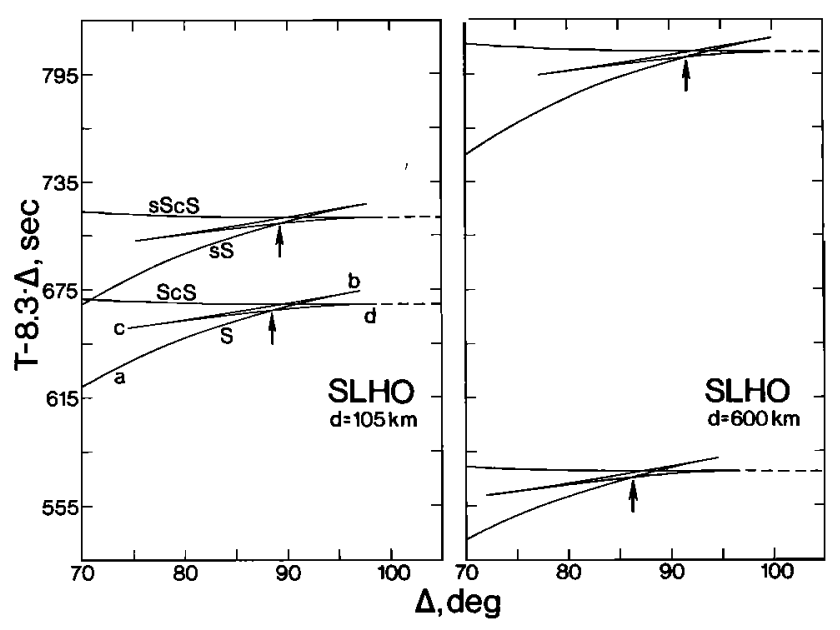

Fig. 2. Travel time branches for direct $\mathbf{S}$ and $\mathrm{sS}$ energy for the discontinuity model SLHO [Lay and Helmberger, 1983] for two source depths. The arrows mark the crossover of the ab branches (energy turning above the $2.75 \%$ discontinuity $280 \mathrm{~km}$ above the core) and the cd branches (energy turning below the discontinuity.)

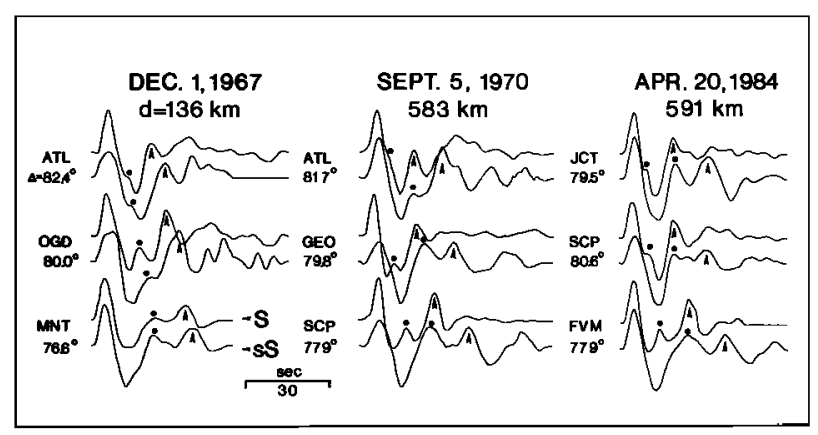

Fig. 3.. Long period tangential component $S$ and sS wavetrains from Sea of Okhotsk events recorded in North America. The arrows indicate the core-reflections and the dots denote the $\mathrm{cd}$ branch arrivals.

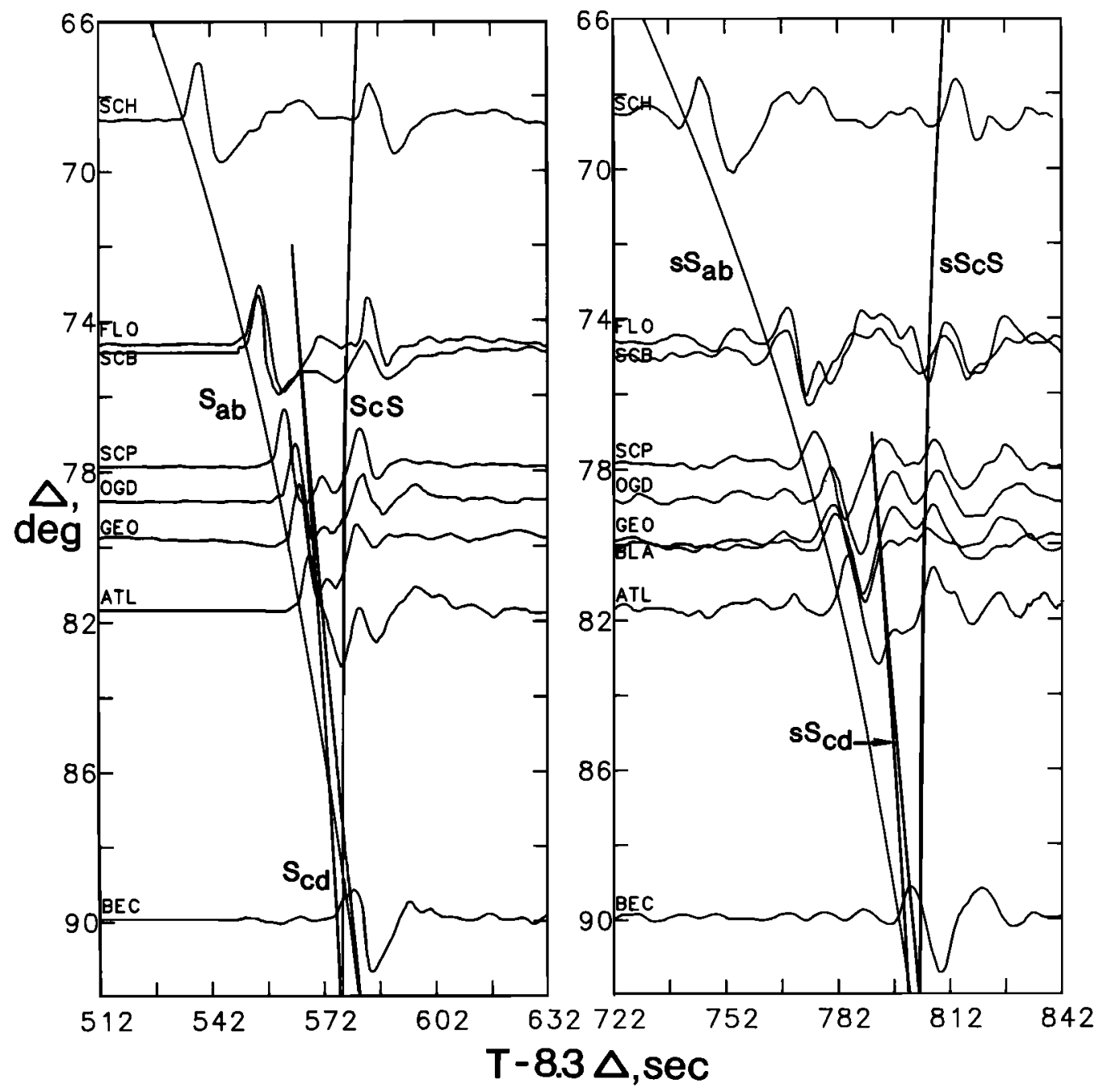

Fig. 4. Profiles of long-period tangential component $S$ and sS waves from the September 5, 1970 Sea of Okhotsk event $(d=583 \mathrm{~km})$. The travel time curves are for model SLHO. 


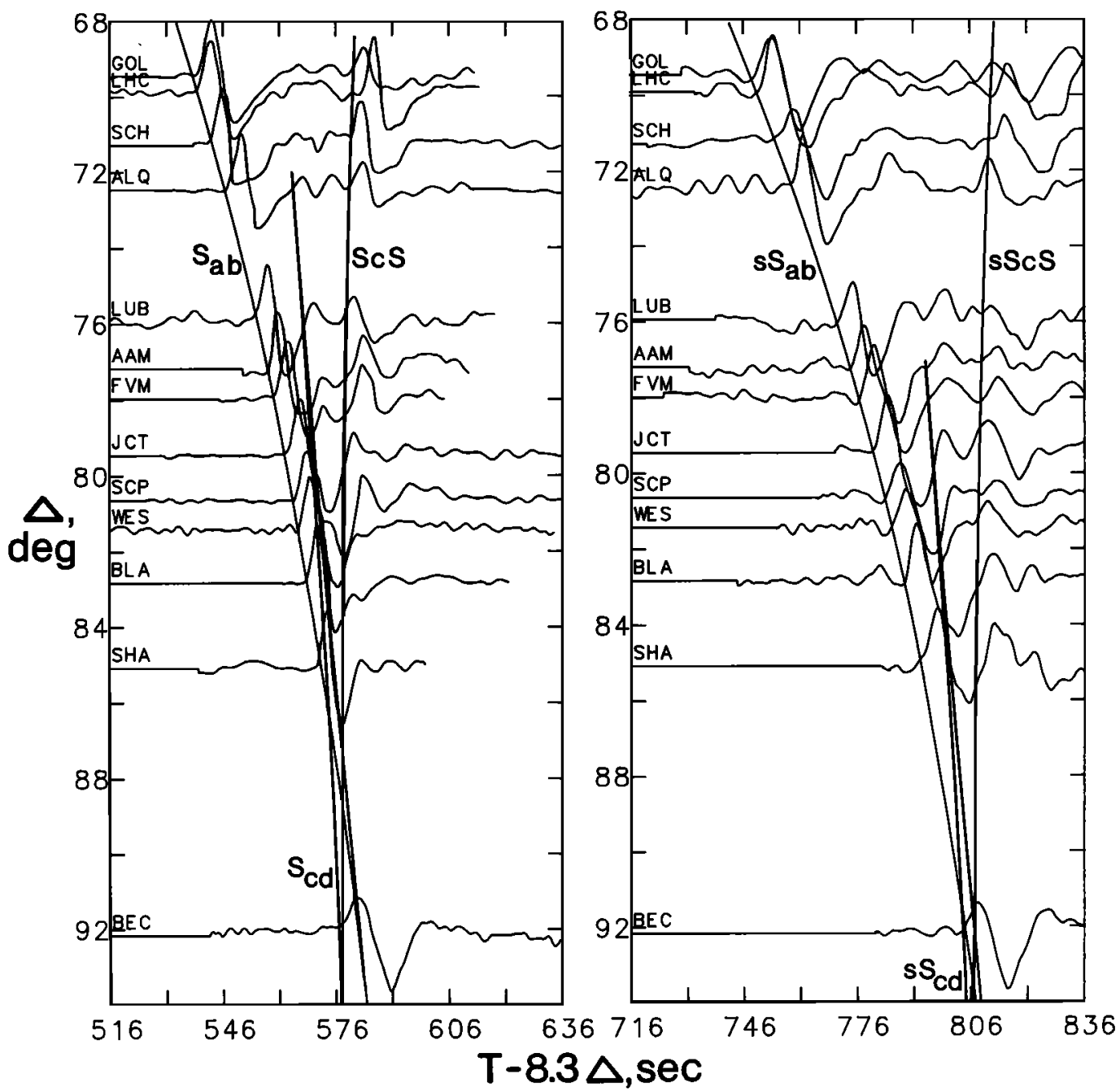

Fig. 5. Profiles of long-period tangential component S and sS waves from the April 20, 1984 Sea of Okhotsk event $(\mathrm{d}=591 \mathrm{~km})$. The travel time curves are for model SLHO.

event, the $S$ and sS wavetrains should be very similar, allowing for differences in radiation pattern and the additional attenuation expected for the sS paths. However, the discontinuity model predicts that the $S$ and $\mathrm{sS}$ waveforms at a given distance from a $600 \mathrm{~km}$ deep event should be very different because the relative timing of the triplications and core-reflections will shift. A smooth lower mantle model such as the JB model predicts only an increase in the time separation sScS-sS compared to ScS-S, with no additional arrivals.

The third attribute of the sS phases is that near the source the raypaths differ substantially from the direct $S$ rays; thus any multipathing or diffraction from deep slab structure should not affect the phases equally. In addition, at a given station, the $S$ and sS phases arrive with nearly identical angles of incidence. Thus, any receiver reverberations should have the same relative timing in the $\mathrm{S}$ and $\mathrm{sS}$ waveforms. For the small earthquake sources $\left(m_{b}=5.7-6.0\right)$ used in this study, source complexity is usually rather unimportant, and any multiple rupture characteristics should have common signatures in the $\mathbf{S}$ and $\mathrm{s} S$ waveforms, further constraining possible explanations of any waveform complexities.

Figure 3 demonstrates that long-period SH component record- ings of $\mathbf{S}$ and sS do exhibit the expected behavior with varying source depth. The $S$ and $\mathrm{sS}$ signals at the same station are aligned on the first arrival. The core-reflected energy, indicated by the arrows, shows only a $4 \mathrm{~s}$ relative shift for the $136 \mathrm{~km}$ deep event, but a $12 \mathrm{~s}$ shift for the deep events. The dots indicate a systematic arrival between the first arrival and the core reflection. For the $136 \mathrm{~km}$ deep event this arrival shifts in time by $3 \mathrm{~s}$ between the $\mathrm{S}$ and sS waveforms, but for the deep events this arrival shifts by about $10 \mathrm{~s}$. If this additional complexity were due to source or receiver reverberations, it should have no relative shift between the $S$ and sS branches. Also note that at a given station, such as SCP or ATL, recordings at different distances show systematic changes in the timing of the intermediate arrival, further precluding a receiver complexity explanation.

Profiles of direct $\mathrm{S}$ and sS wavetrains from a deep focus event in the Sea of Okhotsk (September 5, 1970, d=583 km) recorded across North America are shown in Figure 4. The paths sample the deep mantle beneath Alaska. Lay and Helmberger [1983] modeled the direct $\mathrm{S}$ phases from this event, finding that model SLHO matches the waveforms well. The travel time curve for model SLHO is superimposed on both the S and SS data to test whether this discontinuity model predicts the relative shift of the 


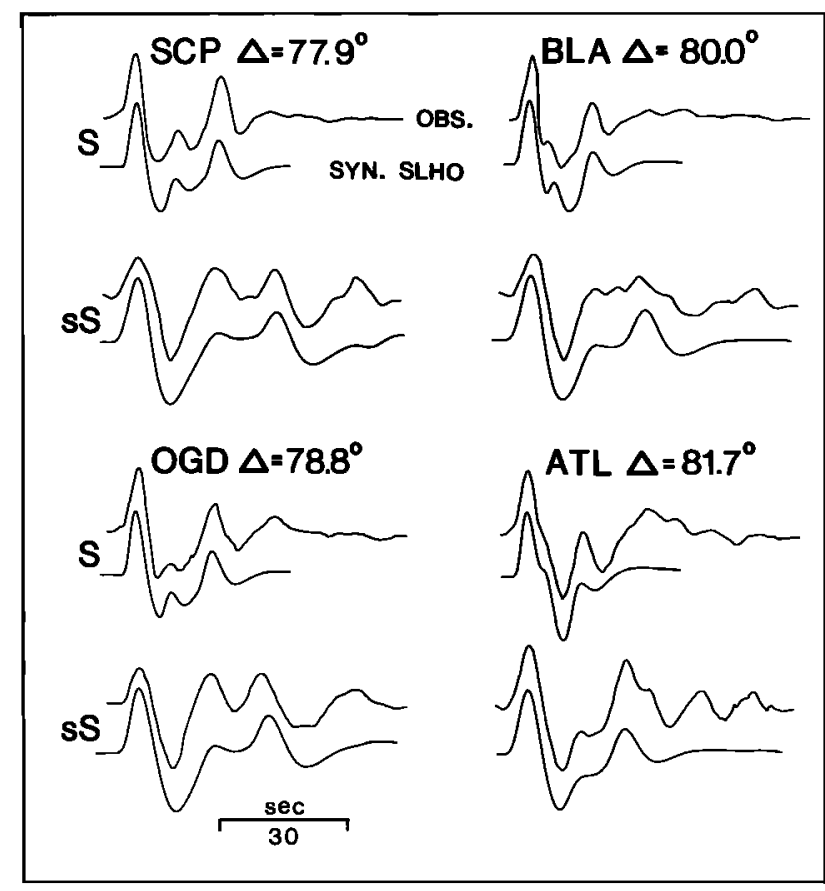

Fig. 6. Observed and synthetic long period tangential component $\mathrm{S}$ and sS waveforms for the September 5, 1970 event. The synthetics are for model SLHO.

sS waveforms expected for this deep event. The triplication crossover distance shifts between the branches by about $5^{\circ}$; thus an sS waveform near $82^{\circ}$ (ATL) should resemble a direct $S$ waveform near $77^{\circ}$ (SCP). Note the consistency of the arrivals between $\mathrm{S}$ and $\mathrm{ScS}$ in the range $74^{\circ}$ to $90^{\circ}$. SLHO accurately predicts the presence of the arrival between $\mathrm{SS}$ and $\mathrm{sScS}$ as well (the sScd branch), and its systematic moveout with distance.

Figure 5 presents a similar comparison of $\mathrm{S}$ and $\mathrm{sS}$ waveforms for a Sea of Okhotsk event (April 20, 1984, d=591 km) that was not previously modeled. Model SLHO accurately predicts the clear arrivals between $\mathrm{S}$ and $\mathrm{ScS}$ as well as those between $\mathrm{SS}$ and $\mathrm{sScS}$. The shift of the waveforms at a common station (for example, JCT) are clearly consistent with the moveout predicted for the triplication. At distances before the cusp of the sScd branch, the waveform complexity disappears. This behavior is predicted by model SLHO, for the reflection coefficient from the discontinuity goes to zero in this range.

Synthetic waveforms confirm the results of the travel time comparison, as shown in Figure 6. Generalized ray theory synthetics for model SLHO match the relative timing and amplitude of the triplication arrivals and core-reflections quite well. Note that the first arrival of the observed sS waveforms tend to have a strong overshoot, which is not accurately modeled. This appears to be a phase shift resulting from the surface reflection interactions. In general, these observations provide strong support for the discontinuity model.

\section{Discussion}

Many additional sS wavetrains have been found to be compatible with the lower mantle shear velocity discontinuity model beneath Alaska proposed by Lay and Helmberger [1983]. The robust waveform shifts and geometry of the S-sS comparison provide a strong test of this model. These data are not sensitive to the slight modifications of SLHO that Lay [1985] has proposed to satisfy diffracted $\mathbf{S}$ data. It is reasonable to conclude that a lower mantle discontinuity does exist at the top of D" beneath Alaska. Evidence for a similar discontinuity has been found in other regions as well [Lay and Helmberger, 1983; Young and Lay, 1986], but it is not definitely established whether this is a global feature. Given strong lateral heterogeneities in D", it is unclear whether we are imaging a localized compositional heterogeneity or a radially symmetric layer embedded within the lateral variations. Further investigations are needed to address the global extent of this structure.

Acknowledgments.This research was supported by the Exxon Education Foundation, a Shell Faculty Career Initiation Grant and NSF grants EAR-8407792 and EAR-8451715.

\section{References}

Clayton, R. W., and R. P. Comer, A tomographic analysis of mantle heterogeneities from body wave travel time data (abstract), Eos, Trans. AGU, 64, 776, 1983.

Doornbos, D. J., Present seismic evidence for a boundary layer at the base of the mantle, J. Geophys. Res. , 88, 3498-3505, 1983.

Doornbos, D. J., S. Spiliopoulos, and F. D. Stacey, Seismological properties of D" and the structure of a thermal boundary layer, Phys. Earth Planet. Interiors, 41, 225-239, 1986.

Dziewonski, A. M., Mapping the lower mantle: Determination of lateral heterogeneity in P velocity up to degree and order 6 , J. Geophys. Res., 89, 5929-5952, 1984.

Jeanloz, R., and F. M. Richter, Convection, composition and thermal state of the lower mantle, J. Geophys. Res., 84, 5497-5504, 1979.

Lay, T., Analysis of diffracted $\mathrm{S}$ waves traversing a region with a lower mantle shear velocity discontinuity (abstract), Eos, Trans. $A G U, 66,310,1985$.

Lay, T., and D. V. Helmberger, A lower mantle S-wave triplication and the shear velocity structure of D", Geophys. J. Roy. Astron. Soc., 75, 799-838, 1983.

Loper, D. E., The dynamical structures of D" and deep plumes in a non-Newtonian mantle, Phys. Earth Planet. Interiors, 34, 57-67, 1984.

Schlittenhardt, J., J. Schweitzer, and G. Müller, Evidence against a discontinuity at the top of D", Geophys. J. R. Astron. Soc., 81, 295-306, 1985.

Young, C. J., and T. Lay, Evidence for a shear velocity discontinuity in the lowermost mantle beneath India and the Indian Ocean, Eos, Trans. AGU, 67, 311-312, 1986.

Wright, C., K. J. Muirhead, and A. E. Dixon, The P wave velocity structure near the base of the mantle, J. Geophys. Res. , 90, 623-634, 1985.

T. Lay, Department of Geological Sciences, University of Michigan, Ann Arbor, MI 48109.

(Received May 27, 1986; Accepted June 9, 1986) 